gramme, and in particular the grouping together of all forms, which, like the resultant, are reducible to zero by the aid of given equations, under the class name of an algebraic modulus. In his Festschrift and the later expository papers of his pupils are proposed methods for testing any given system for its character, whether general, or special of the first sort (loci with a curve in common), or of the second or higher sort (loci with a surface, etc., in common). The expansion of this body of doctrine or abstract theory into a concrete geometry with fulness of examples remains a task, not all deductive but largely creative, for coming decades or generations.

Not the possession of eliminants actually calculated by Bézout's deservedly famous scheme is needful for the geometer, but the knowledge of the conditions under which such an eliminant will exist, and what conditions will modify it. So with regard to the more far-reaching scheme of Kronecker ; it is ultimately, perhaps, not the full elaboration of particular examples as such, that we wish to have, but a precise knowledge of how the relative operations could be executed in finite time, and a precise formulation of conditions that would modify or influence the result of those operations. Which is of greater value, the logic or the concrete object to which it is applied? Let everyone decide when both are in his possession!

\title{
ON THE REPRESENTATION OF NUMBERS BY MODULAR FORMS.
}

BY PROFESSOR L. E. DICKSON.

(Read before the Chicago Section of the American Mathematical Society, January 2, 1909.)

1. For any field $F$ in which there is an irreducible equation $f(\rho)=0$ of degree $m$, the norm of

$$
x_{0}+x_{1} \rho+x_{2} \rho^{2}+\cdots+x_{m-1} \rho^{m-1}
$$

is a form of degree $m$ in $m$ variables which vanishes for no set of values $x_{i}$ in the field $F$, other than the set in which every $x_{i}=0$. For a finite field it seems to be true that every form of degree $m$ in $m+1$ variables vanishes for values, not all zero, in the field. For $m=2$ and $m=3$ this theorem is 
established in $\S \S 2,3$. The corresponding theorem does not hold in general for infinite fields. But A. Meyer* has shown that any indefinite quadratic form in five variables vanishes for integral values, not all zero, of the variables.

Modular forms which represent only squares have been investigated $\dagger$ at length by the writer; those which represent cubes exclusively are considered in $\S \S 4-12$.

2. Any ternary quadratic form in the $G F\left[p^{n}\right], p>2$, can be transformed into $q=a x^{2}+b y^{2}+c z^{2}$. The number of sets of solutions of $q=0$ in the field has been determined; $\$$ in particular, this number exceeds unity. The latter fact may be proved very simply as follows. Since the proposition is evident when $a=0$, we may take $a=-1, c \neq 0$. Let $z=t y$. Then the question is whether or not $\left(b+c t^{2}\right) y^{2}-x^{2}$ vanishes in the field for $x$ and $y$ not both zero. If not, $b+c t^{2}$ would be a not-square for every $t$. But there are only $\frac{1}{2}\left(p^{n}-1\right)$ notsquares and $\frac{1}{2}\left(p^{n}+1\right)$ squares $t^{2}$, including zero. Hence not every one of the $\frac{1}{2}\left(p^{n}+1\right)$ values of $b+c t^{2}$ is a not-square.

3. Let $Q$ be a quaternary cubic form with coefficients in the $G F\left[p^{n}\right], p>3$, which vanishes for no set of values in the field for $x, y, z, w$, except the set $x=0, \cdots, w=0$. The coefficient of $x^{3}$ is not zero and may be taken to be unity. By adding to $x$ a linear function of $y, z, w$, we may delete the terms $x^{2} y, x^{2} z, x^{2} w$. The coefficient of $x$ may be transformed into $d y^{2}+\lambda z^{2}+\mu w^{2}$. Then $Q$ becomes

$$
\begin{aligned}
Q_{1}=x^{3}+x\left(d y^{2}\right. & \left.+\lambda z^{2}+\mu w^{2}\right)+g y^{3}+A y^{2} z+B y^{2} w+C y z^{2} \\
& +D y w^{2}+E y z w+F z^{3}+G z^{2} w+H z w^{2} w+R^{3} .
\end{aligned}
$$

Let $w=t z$. Then $Q_{1}$ becomes a ternary cubic form which may be identified with that on page 161 of volume 14 of the BuLLE IIN, by setting $a=1, b=c=f=0$,

$$
\begin{gathered}
e=\lambda+\mu t^{2}, \quad h=A+B t, \quad k=C+E t+D t^{2}, \\
l=F+G t+H t^{2}+R t^{3} .
\end{gathered}
$$

Hence the relations at the top of page 165 must hold for every $t$. From $d h \neq 0$, we conclude that $d \neq 0, B=0$. Then from the coefficient of $t^{3}$ in $e h+3 d l=0$, we have $R=0$. Hence $Q_{1}$ vanishes for $x=y=z=0, w=1$, contrary to hypothesis.

* Bachmann, Zahlentheorie, IV, p. 266, p. 553.

† Transactions Amer. Math. Society, vol. 10 (1909), pp. 109-122.

$\ddagger$ The writer's Linear Groups, pp. $47,48$. 
4. In investigating forms which represent exclusively cubes* in the $G F\left[p^{n}\right]$, we shall assume that $p^{n}$ is of the form $3 k+1$. For if $p^{n}-1$ were prime to 3 , every element $\neq 0$ of the field would equal a cube in the field, and the question would be without content.

Let $a x^{d}+\cdots$ represent cubes exclusively. In view of the values $x=1, y=0, \cdots, a$ must be a cube. Hence we may assume that the coefficient of $x^{d}$ is unity. Further $d$ must be a multiple of 3. For, if $d$ is prime to 3 , the form represents the not-cube $v^{d}$ when $x$ equals a not-cube $v, y=0, \ldots$ We may therefore restrict our attention to forms $x^{3 l}+\cdots$ in the $G F\left[p^{n}\right]$, $p^{n}=3 k+1$.

5. Theorem. No cubic form represents cubes exclusively.

It suffices to prove the theorem for irreducible binary cubic forms $C$ in the $G F\left[p^{n}\right], p^{n}=3 k+1$. Then $C(x, 1)=0$ has the roots $\rho, \rho^{p^{n}}, \rho^{p^{2 n}}$ in the $G F\left[p^{3 n}\right]$, so that

$$
C=(x-\rho y)^{t}, \quad t=1+p^{n}+p^{2 n} .
$$

If $C$ represent only cubes, $C^{\left(p^{n-1}\right) / 3}=1$. Hence the power $\frac{1}{3}\left(p^{3 n}-1\right)$ of $x-\rho y$ equals unity, so that $x-\rho y$ is a cube in the $G F\left[p^{3 n}\right]$ for every $x$ and $y$, not both zero, in the $G F\left[p^{n}\right]$. Any non-vanishing element $\mu$ of the latter is a cube in the $G F\left[p^{3 n}\right]$. Hence

$$
\mu(a-\rho) /(b-\rho) \quad(a \neq b)
$$

furnishes $p^{n}\left(p^{n}-1\right)^{2}$ distinct cubes in the $G F\left[p^{3 n}\right]$, a number exceeding the total number $\frac{1}{3}\left(p^{3 n}-1\right)$ of the cubes.

6. In the $G F\left[p^{n}\right], p^{n}=3 k+1$, let $S(x, y)$ be a sextic representing only cubes. Since $S$ is the product of $S\left(x y^{-1}, 1\right)$ by $y^{6}$, it suffices to require that $S(x, 1)$ shall represent only cubes.

First, let $p=2$, so that $n$ is even, $n=2 m$. Set

$$
S=\sum_{i=0}^{6} a_{i} x^{i} \quad\left(a_{6}=1\right) .
$$

Now $S^{(4 m-1) / 3}=1$ since $S$ shall represent only cubes in the $G F\left[4^{m}\right]$. The exponent equals $\sum_{j=0}^{m-1} 4^{j}$. Hence, for every $x$ in the field,

\footnotetext{
* A non-vanishing element equal to the cube of an element of the field.
} 


$$
\prod_{j=0}^{m-1}\left(\sum_{i=0}^{6} \alpha_{i}^{4^{j}} x^{i} \cdot 4^{j}\right)=1
$$

The powers of $x$ may be reduced by $x^{4^{m}}=x$. Hence, for $1 \leqq e \leqq 4^{m}-1$,

$$
\sum a_{i_{0}} a_{i 1}^{4} a_{i_{2}}^{42} \cdots a_{i_{m-1}}^{4 m-1}=0,
$$

the sum extending over all $*$ integers $0 \leqq i \leqq 6$ for which (3) $E=i_{0}+4 i_{1}+4^{2} i_{2}+\cdots+4^{m-1} i_{m-1} \equiv e\left(\bmod 4^{m}-1\right)$.

It is found to be advantageous to consider the higher values of $e$.

For $e=4^{m}-1$, we have $E=2 e$ (whence each $i=6$ ) or $E=e$. But

$$
4^{m}-1=3+3 \cdot 4+3 \cdot 4^{2}+\cdots+3 \cdot 4^{m-1} .
$$

Hence $E=e$ gives $i_{0} \equiv 3(\bmod 4)$, whence $i_{0}=3, i_{1}=3$; etc. Since $a_{6}=1$, (2) becomes

$$
1+a_{3}^{\left(4^{m}-1\right) / 3}=0 .
$$

For $e=4^{m}-2, E=2\left(4^{m}-1\right)-1$ gives $i_{0}=5, i_{j}=6(j \geqq 1)$. For $E=e$, either $i_{0}=2, i_{j}=3(j \geqq 1)$, or $i_{0}=6$. In the latter case, $\frac{1}{4}(E-e)$ gives

$1+i_{1}-3+4\left(i_{2}-3\right)+4^{2}\left(i_{3}-3\right)+\cdots+4^{m-2}\left(i_{m-1}-3\right)=0$.

Thus $i_{1} \equiv 2(\bmod 4)$. For $i_{1}=2$, each $i_{j}=3(j \geqq 2)$. For $i_{1}=6$,

$$
1+i_{2}-3+4\left(i_{3}-3\right)+\cdots+4^{m-3}\left(i_{m-1}-3\right)=0 .
$$

Either $i_{2}=2, i_{j}=3(j \geqq 3)$, or $i_{2}=6$ and we proceed as before. Thus

$$
a_{5}+a_{2} a_{3}^{c_{1}}+a_{2}^{4} a_{3}^{c_{2}}+a_{2}^{42} a_{3}^{c_{3}}+\cdots a_{2}^{4 m-1} a_{3}^{c_{m}}=0
$$

$$
\left[c_{r}=\frac{1}{3}\left(4^{m}-4^{r}\right)\right] \text {. }
$$

Similarly, for $\dagger e=4^{m}-t, t=3,4,6,7,8$, we get

$$
\begin{gathered}
a_{4}+a_{1} a_{3}^{c_{1}}+a_{5}\left(a_{2}^{4} a_{3}^{c_{2}}+a_{2}^{42} a_{3}^{c_{3}}+\cdots+a_{2}^{4 m-1} a_{3}^{c_{m}}\right)=0, \\
a_{3}+a_{0} a_{3}^{c_{1}}+a_{4}\left(a_{2}^{4} a_{3}^{c_{2}}+a_{2}^{42} a_{3}^{c_{3}}+\cdots\right)=0 \\
a_{1}+a_{5}^{21}+a_{2}\left(a_{2}^{4} a_{3}^{c_{2}}+a_{2}^{4^{2}} a_{3}^{c_{3}}+\cdots\right) \\
+a_{1}^{4} a_{3}^{c_{2}}+a_{5}^{4}\left(a_{2}^{4^{2}} a_{3}^{c_{3}}+a_{2}^{4^{3}} a_{3}^{c_{4}}+\cdots\right)=0,
\end{gathered}
$$

* Except the set in which each $i=0$, when $e=4^{m}-1$.

$\uparrow$ The result for $e=4^{m}-5$ is evidently the fourth power of (6). 


$$
\begin{aligned}
& a_{0}+a_{4} a_{5}^{4}+a_{1}\left(a_{2}^{4} a_{3}^{c_{2}}+\cdots\right) \\
& +a_{5} a_{1}^{4} a_{3}^{c_{2}}+a_{5}^{5}\left(a_{2}^{42} a_{3}^{c_{3}}+\cdots\right)=0, \\
& \text { (11) } a_{3} a_{5}^{4}+a_{0}\left(a_{2}^{4} a_{3}^{c_{2}}+\cdots\right)+a_{4} a_{1}^{4} a_{3}^{c_{2}}+a_{4} a_{5}^{4}\left(a_{2}^{42} a_{3}^{c_{3}}+\cdots\right)=0 \text {. }
\end{aligned}
$$

For $m=1$ the last three conditions do not occur.

First, let $a_{5}=0 . \quad$ Replacing $x$ by $x+a_{4}^{3 / 2} y$, we have $a_{5}=0$, $a_{4}=0$. By (5), $a_{3}$ is a cube in the field. By multiplying $y$ by $a_{3}^{-1 / 3}$, we have $a_{3}=1$. Then $a_{1}=0$ by (7). By (6),

$$
a_{2}+a_{2}^{4}+a_{2}^{4^{2}}+\cdots+a_{2}^{4 m-1}=0,
$$

so that $a_{2}=0$ if $m=1$. For $m>1,(9)$ and (10) give $a_{2}=0$, $a_{0}=0$, so that the sextic vanishes for $x=0$. Hence $m=1$, and

(12) $S=x^{6}+x^{3} y^{3}+y^{6}=\left[x^{3}+\sigma y^{3}\right]\left[x^{3}+(\sigma+1) y^{3}\right]$,

where $\sigma^{2} \equiv \sigma+1(\bmod 2)$ defines the $G F[4]$.

Next, for $a_{5} \neq 0$, we multiply $y$ and make $a_{5}=1$. We multiply (6) by $a_{3}$ and add the fourth power of (6). We obtain

$$
1+a_{2}+a_{3}=a_{2} a_{3}^{c_{1}+1}=a_{2},
$$

by (5). Hence $a_{3}=1$, so that (6) becomes

$$
1+a_{2}+a_{2}^{4}+a_{2}^{42}+\cdots+a_{2}^{4 m-1}=0 .
$$

In view of the latter, (7) and (8) give

$$
a_{1}=a_{4}+a_{2}+1, \quad a_{0}=1+a_{4}\left(a_{2}+1\right) .
$$

If $m=1, a_{2}=1$ by $\left(6^{\prime}\right)$, so that *

$$
S=x^{6}+x^{5} y+a_{4} x^{4} y^{2}+x^{3} y^{3}+x^{2} y^{4}+a_{4} x y^{5}+y^{6} .
$$

If $a_{2}^{4}+a_{4}=1$, we have (13). If $a_{4}=0$, we interchange $x$ with $y$ and are led to the earlier case $\alpha_{5}=0$. If $a_{4}=1$, we replace $x$ by $x+\sigma y$, where $\sigma^{2}+\sigma+1=0$ and obtain a sextic $S$ with $a_{4}=0$.

If $m>1$, we apply $\left(6^{\prime}\right)$ to (9) and obtain

$$
a_{1}+1+a_{2}\left(a_{2}+1\right)+a_{1}^{4}+a_{2}^{4}+a_{2}+1=0 .
$$

Eliminating $a_{1}$ and $a_{0}$ by $\left(7^{\prime}\right)$, we get

$$
a_{2}^{2}+a_{2}=a_{4}+a_{4}^{4} \text {. }
$$

* In the simple case $m=1$, we may also proceed directly with (1), which must reduce to unity (the only cube) for every $x$. 
Condition (10) likewise leads to $\left(9^{\prime}\right)$. But (11) gives

$$
a_{2}+a_{2}^{2} a_{4}+a_{4}+a_{4}^{5}+a_{2} a_{4}=0 .
$$

By $\left(9^{\prime}\right)$ and $\left(11^{\prime}\right)$, we get

$$
a_{2}=a_{4}+a_{4}^{2} \text {. }
$$

By $\left(7^{\prime}\right), a_{1}=a_{4}^{2}+1, a_{0}=a_{4}^{3}+a_{4}^{2}+a_{4}+1$. But $a_{3}=a_{5}=1$. Hence

$$
S=\left[x^{2}+x y+\left(a_{4}+1\right) y^{2}\right]^{3} .
$$

Theorem. In the $G F^{\prime}\left[2^{n}\right]$, n even, every binary sextic which represents only cubes is formally a perfect cube, except for the case $n=2$, when there is an additional type (12), the product of two irreducible cubic forms.

7. For $p^{n}=3 k+1, p>2$, we may remove the term $x^{5} y$ from the binary sextic $S$. Hence it suffices to investigate

$$
S=z^{6}+b z^{4}+c z^{3}+d z^{2}+e z+f \quad(f=\text { cube }) .
$$

For $p^{n}=7$, the cubes are \pm 1 . We require that $S^{2} \equiv 1$ for every $z$ making $z^{6} \equiv 1(\bmod 7)$. First, let $f=-1$. Then

requires that

$$
\left(b z^{4}+c z^{3}+d z^{2}+e z\right)^{2} \equiv 1
$$

$b e+c d \equiv 0, \quad d^{2}+2 c e \equiv 0, \quad d e \equiv 0, \quad b^{2}+e^{2} \equiv 0$,

$$
b c \equiv 0, \quad c^{2}+2 b d \equiv 1 .
$$

Hence $S=z^{6} \pm z^{3}-1$. Multiplying $z$ by \pm 1 , we get

(15) $z^{6}+z^{3}-1=\left(z^{2}-z+3\right)\left(z^{2}-2 z-2\right)\left(z^{2}+3 z-1\right)$.

Next, let $f=1$. Then $\left(b z^{4}+\cdots+2\right)^{2}=1$ gives

$$
\begin{aligned}
b e+c d \equiv d^{2}+2 c e+4 b \equiv 2 c+d e \equiv b^{2}+e^{2}+4 d & \\
& \equiv b c+2 e \equiv c^{2}+2 b d+3 \equiv 0 .
\end{aligned}
$$

If $e \equiv 0$, then $c \equiv 0, d \equiv-2 b^{2}, b^{3} \equiv-1$. Multiplying $z$ by $3 b^{-1}$, we have $b=3$. Hence

$$
S=\left(z^{2}+1\right)^{3} \text {. }
$$

If $e \neq 0$ we multiply $y$ by $e$ and have $e=1$. Then the first three conditions give

$$
b \equiv 2 c^{2}, \quad c^{2} \equiv c, \quad d \equiv 5 c .
$$


The fifth gives $c^{3} \equiv-1$, in contradiction with $c^{2} \equiv c$.

Any sextic $a x^{6}+\cdots$ which represents only cubes, modulo 7, cannot equal -1 for every $x$ and $y$, not both zero, and hence must take the value +1 . Hence it can be transformed into $x^{6}+\cdots$.

Theonem. Every binary sextic which represents only cubes, modulo 7, can be transformed into

$$
\left(x^{2}+y^{2}\right)^{3} \quad \text { or } \quad x^{6}+x^{3} y^{3}-y^{6} .
$$

8. Let $\lambda=z^{6}+b z^{4}+d z^{2}+f, \mu=c z^{3}+e z$. Then $\lambda+\mu$ and $\lambda-\mu$ must be cubes for every $z$. For $p^{n}=13,(\lambda \pm \mu)^{4} \equiv 1$, whence

$$
\lambda \equiv 0, \mu^{4} \equiv 1 ; \mu \equiv 0, \lambda^{4} \equiv 1 ; \lambda^{2}+\mu^{2} \equiv 0, \lambda^{4} \equiv \mu^{4} \equiv 3
$$

$(\bmod 13)$.

Hence for every $z$,

$$
\mu\left(\mu^{4}-1\right)\left(\mu^{4}-3\right) \equiv 0(\bmod 13) .
$$

If $e \equiv 0, \mu=c z^{3}$, and (18) gives

$$
c\left(c^{4}-1\right)\left(c^{4}-3\right) \equiv 0(\bmod .13) .
$$

If $c^{4} \equiv 1$, then $\mu^{4} \equiv 1$ for $z \neq 0$, so that, by (17), $\lambda \equiv 0$ for every $z \neq 0$, contrary to the degree of $\lambda$. If $e^{4} \equiv 3$, then $\mu^{4} \equiv 3, \lambda^{2}+\mu^{2} \equiv 0$ for $z \neq 0$; but the resulting conditions give $b \equiv d \equiv 0, c^{2} \equiv \pm 3$, contrary to $c^{4} \equiv 3$.

For the troublesome case $c=e=0$, we set

$$
\lambda=z^{6}+d z^{2}, \quad \mu=b z^{4}+f,
$$

so that $S=\lambda+\mu$ is a cube. Replacing $z$ by $5 z$, we see that $-\lambda+\mu$ is a cube for every $z$. Hence one of the three cases (17) must hold. Now $\mu\left(\mu^{4}-3\right)$ involves only powers of $z^{4}$ and hence, for $z \neq 0$, equals a function of degree 8. Thus at least four values $\neq 0$ of $z$ must give rise to the first case (17). Hence $\lambda=0$ has four roots $z \neq 0$, so that $d^{3}=-1$. Multiplying $y$ by $d^{-1}$, we may set $d=-1$. Thus $\lambda=z^{2}\left(z^{4}-1\right)$. When $\lambda \neq 0$, we have $z^{4}=3$ or 9 , viz.,

$$
\begin{aligned}
& z^{4}=3, \quad \lambda=2 z^{2}, \quad \lambda^{2}=-1, \quad \lambda^{4}=1 ; \\
& z^{4}=9, \quad \lambda=8 z^{2}, \quad \lambda^{2}=4, \quad \lambda^{4}=3,
\end{aligned}
$$

thus corresponding to the respective cases (17). Thus if $z^{4}=3$, then $\mu=0$, whence $3 b=-f$. For $z^{4}=9$, then $\mu^{2}+4=0$, $f^{2}=-1$. Hence $S=\left(z^{2} \pm 2\right)^{3}$. 
Finally, let $e \neq 0$. Since every integer is a fifth power modulo 13, we may multiply $y$ and make $e=1$. Then (18) holds for $\mu=c z^{3}+z$. For $z \neq 0$,

$$
\begin{aligned}
& \mu^{8}-4 \mu^{4}+3=\left(8 c^{7}-3 c^{3}+8 c\right) z^{10}+\left(2 c^{6}+2 c^{2}+1\right) z^{8} \\
& +\left(4 c^{5}-3 c\right) z^{6}+\left(5 c^{4}-4\right) z^{4}+4 c^{3} z^{2}+c^{8}-4 c^{4}+2 c^{2}+3 .
\end{aligned}
$$

In its product by $c z^{2}+1$, the coefficients of $z^{4}$ and $z^{6}$ are

$$
9 c^{4}-4,9 c^{5}-7 c,
$$

which do not both vanish modulo 13 .

Hence the sextics which represent only cubes modulo 13 have been reduced to the product of $\left(x^{2} \neq 2 y^{2}\right)^{3}$ by a cube $l^{3}$. But $l\left(x^{2} \pm 2 y^{2}\right)$ can be transformed into $x^{2}+2 y^{2}$.

Theorem. Every sextic which represents only cubes modulo 13 is a perfect cube which may be transformed into $\left(x^{2}+2 y^{2}\right)^{3}$.

9. For the next two values 19 and 25 of $p^{n}=3 k+1$, I find that any binary sextic $S$ which represents only cubes equals the cube of a quadratic form. For $p^{n}=5^{2}$, this result follows readily by requiring that $S^{2}$ shall equal its fifth power; by the coefficients of $z^{21}, z^{16}, z^{11}, z^{4}$, we get $S=\left(z^{2}+2 b\right)^{3}$.

For $p^{n}=11^{2}, S^{4}$ must equal its 11 th power. By the coefficients of $z^{111}, z^{100}, z^{89}, z^{78}$, we get $S=\left(z^{2}+4 b\right)^{3}$.

It seems probable that, for $p^{n}=3 k+1>7$, every binary sextic which represents only cubes is formally a pertect cube. Although certain general considerations point to the validity of this conjecture, I have not effected a complete proof.

10. A sextic on three or more variables cannot represent exclusively cubes in a field of order $p^{n}=3 k+1$ for which the binary sextics representing only cubes are formally perfect cubes. It suffices to prove the theorem for ternary sextics $T$. For $* p>2$, we may delete the terms $x^{5} y, x^{5} z, y^{5} z$. Then the terms free of $z$ are $\left(x^{2}-v y^{2}\right)^{3}$, where $v$ is a not-square. When $z$ is multiplied by a suitable constant, the terms free of $y$ are $\left(x^{2}-v z^{2}\right)^{3}$. Then the terms free of $x$ are $-v^{3}\left(y^{2}+\epsilon z^{2}\right)^{3}$, where $\epsilon^{3}=1$. Since $\epsilon$ equals the square of $\epsilon^{2}$, and $y^{2}+\epsilon z^{2}$ is irreducible, -1 must be a not-square. Hence we may set $v=-1$. Hence

$$
\begin{aligned}
T= & x^{6}+y^{6}+z^{6} \\
& +3\left(x^{4} y^{2}+x^{2} y^{4}+x^{4} z^{2}+x^{2} z^{4}+\epsilon y^{4} z^{2}+\epsilon^{2} y^{2} z^{4}\right)+x y z \phi,
\end{aligned}
$$

* By a different argument, I have proved that there is no tenary sextic in the $G F^{\prime}\left[2^{2 m}\right]$ which represents only cubes. 


$$
\begin{aligned}
\phi=A x^{3}+B y^{3}+C z^{3}+ & D x^{2} y+E x^{2} z+F x y^{2} \\
& +G x z^{2}+K y^{2} z+I y z^{2}+M x y z .
\end{aligned}
$$

Set $x=\lambda y$. Then, for $\lambda$ arbitrary in the field,

$$
f=\left(\lambda^{2}+1\right)^{3} y^{6}+r y^{5} z+s y^{4} z^{2}+\left(G \lambda^{2}+L \lambda\right) y^{3} z^{3}+t y^{2} z^{4}+z^{6}
$$

must be formally a perfect cube in $x, y$. Here

$$
\begin{aligned}
& r=A \lambda^{4}+D \lambda^{3}+F \lambda^{2}+B \lambda, \\
& s=3 \lambda^{4}+E \lambda^{3}+M \lambda^{2}+K \lambda+3 \varepsilon, \\
& t=3 \lambda^{2}+C \lambda+3 \epsilon^{2} .
\end{aligned}
$$

Hence $f=\left[\left(\lambda^{2}+1\right) y^{2}+\omega z^{2}\right]^{3}$, where $\omega^{3}=1$. Thus $r=0, \quad s=3 \omega\left(\lambda^{2}+1\right)^{2}, \quad t=3 \omega^{2}\left(\lambda^{2}+1\right), \quad G \lambda^{2}+L \lambda=0$ for every $\lambda$ in the given field of order $>4$. Hence

$$
\begin{aligned}
A=B=C=D=E=F= & G=K=L=0, \quad M=6, \\
& \omega=\epsilon=1, \quad T=\left(x^{2}+y^{2}+z^{2}\right)^{3} .
\end{aligned}
$$

By $\S 2, T$ vanishes for $x, y, z$, not all zero, in the field.

11. It remains to investigate the ternary sextics $T$ which represent only cubes in the GF[7]. The terms free of $x$ in $T$ will be denoted by $B_{y z}$, those free of $y$ by $B_{x x}$, etc. By $\S 7$, each $B$ is a perfect cube or a product $\pi$ of three quadratic forms. Suppose that one of the $B$ 's, say $B_{x z}$, is of the type $\pi$. Replacing $x$ by $x+r y+s z$, we may delete the terms $x^{5} y$ and $x^{5} z$; then $B_{x z}$ is still of the type $\pi$, and $B_{x y}$ is of its original type. Hence we may set

$$
B_{x z}=z^{6}+x^{3} z^{3}-z^{6} .
$$

Let $T$ become $T^{\prime}$ when $y$ is replaced by $y+\rho z$. The coefficient $c$ of $z^{6}$ in $B_{x z}^{\prime}$ is the value of $B_{y z}$ for $y=\rho, z=1$, and hence is a sextic in $\rho$ with a non-vanishing coefficient for $\rho^{6}$; thus $c \neq-1$ for some value of $\rho$. Since $B_{x y}^{\prime}$ is not of type $\pi$, it is a perfect cube. Also, $B_{x y}^{\prime}=B_{x y}$.

Thus one of the $B$ 's may be assumed to be a perfect cube. With $B_{x y}$ a cube, the same argument shows that we may make also $B_{x z}$ a perfect cube, and that we may set

$$
B_{x z}=\left(x^{2}+z^{2}\right)^{3}, \quad B_{x y}=\left(x^{2}+y^{2}\right)^{3} .
$$

Employing the abbreviation $\phi$, given by (20), we have 
$T=x^{6}+y^{6}+z^{6}+3 x^{4} y^{2}+3 x^{4} z^{2}+3 x^{2} y^{4}+3 x^{2} z^{4}+\sum_{i=1}^{5} c_{i} y^{6-i} z^{i}+x y z \phi$.

Set $y=\lambda z$. Then $T$ becomes

$$
t=x^{6}+r_{2} x^{4} z^{2}+r_{3} x^{3} z^{3}+\cdot+r_{6} z^{6},
$$

$r_{2}=3 \lambda^{2}+A \lambda+3, r_{3}=D \lambda^{2}+E \lambda, r_{4}=3 \lambda^{4}+F \lambda^{3}+M \lambda^{2}+G \lambda+3$,

$r_{5}=B \lambda^{4}+K \lambda^{3}+L \lambda^{2}+C \lambda, r_{6}=\lambda^{6}+c_{1} \lambda^{5}+\cdots+c_{5} \lambda+1$.

Now $\tau=x^{6} \pm x^{3} z^{3}-z^{6}$, viz., is of type $\pi$, if and only if

$$
r_{2} \equiv r_{4} \equiv r_{5} \equiv 0, \quad r_{3}^{2} \equiv 1, \quad r_{6} \equiv-1 \quad(\bmod 7) ;
$$

while $\tau$ is a perfect cube if and only if

$$
r_{3} \equiv r_{5} \equiv 0, \quad r_{4} \equiv 5 r_{2}^{2}, \quad r_{6} \equiv 6 r_{2}^{3} \quad(\bmod 7) .
$$

Since $r_{2} r_{3} \equiv 0$ for every $\lambda, D \equiv E \equiv 0$. Hence (21) is excluded, so that (22) must hold for every $\lambda$. We may therefore remove the term $y^{5} z$ from $T$ and proceed as in $\S 10$. Or we may proceed with (22) and show that

$$
T \equiv\left(x^{2}+y^{2}+z^{2}-2 A y z\right)^{3} .
$$

12. The theorem that there exists no sextic on three or more variables which represents only cubes in a field of order $p^{n}=3 k+1$ has now been established for $p^{n}<31, p^{n}=2^{n}$, and $p^{n}=11^{2}$. Its truth for all values of $p^{n}$ has been proved, subject to the validity of the conjectured theorem of $\S 9$ on binary sextics.

The University of Chicago, October, 1908.

\section{NOTE ON LÜROTH'S TYPE OF PLANE QUARTIC CURVES.}

BY PROFESSOR H. S. WHITE AND MISS KATE G. MILLER.

(Read before the American Mathematical Society, September 6, 1907.)

ONE of the stock examples of the fallacy of constant counting is the equation of a plane quartic, whose fourteen constants equal in number those apparent in the sum of five fourth powers of linear expressions

$$
x_{1}^{4}+x_{2}^{4}+x_{3}^{4}+x_{4}^{4}+x_{5}^{4} .
$$

